\title{
A study on the use of an immersive Virtual Reality store to investigate consumer perceptions and purchase behavior toward non standard fruits and vegetables
}

\author{
Adrien Verhulst* \\ École Centrale de Nantes, AAU UMR CNRS 1563, Nantes, France \\ Cindy Lombart ${ }^{\dagger}$ \\ Audencia Business School, Research Center in Marketing \& \\ Distribution In Situ, Nantes, France
}

\author{
Jean-Marie Normand* \\ École Centrale de Nantes, AAU UMR CNRS 1563, Nantes, France \\ Guillaume Moreau* \\ École Centrale de Nantes, AAU UMR CNRS 1563, Nantes, France
}

\begin{abstract}
In this paper we present an immersive virtual reality user study aimed at investigating how customers perceive and if they would purchase non standard (i.e. misshaped) fruits and vegetables ( $\mathrm{FaVs})$ in supermarkets and hypermarkets. Indeed, food waste is a major issue for the retail sector and a recent trend is to reduce it by selling non-standard goods. An important question for retailers relates to the FaVs' "level of abnormality" that consumers would agree to buy. However, this question cannot be tackled using "classical" marketing techniques that perform user studies within real shops since fresh produce such as FaVs tend to rot rapidly preventing studies to be repeatable or to be run for a long time. In order to overcome those limitations, we created a virtual grocery store with a fresh FaVs section where 142 participants were immersed using an Oculus Rift DK2 HMD. Participants were presented either "normal", "slightly misshaped", "misshaped" or "severely misshaped" FaVs. Results show that participants tend to purchase a similar number of FaVs whatever their deformity. Nevertheless participants' perceptions of the quality of the $\mathrm{FaV}$ depend on the level of abnormality.
\end{abstract}

Index Terms: H.5.2 [Information Interfaces and Presentation]: User Interfaces_Evaluation/Methodology; I.3.7 [Computer Graphics]: Three-Dimensional Graphics and Realism-Virtual Reality

\section{INTRODUCTION}

In 2011, a report from the Food and Agriculture Organization (FAO) of the United Nations [12] stated that roughly one third of the edible food produced in the world for human consumption every year - approximately 1.3 billion tonnes - gets lost or wasted. FaVs, plus roots and tubers (the $\mathrm{FaV}$ commodity group) have the highest wastage rates of any food ranging between 40 and 55\% (e.g., 50\% in North America and Oceania; also 50\% in South and Southeast Asia, $45 \%$ in Europe). For the FaVs commodity group, losses in agricultural production dominate for the aforementioned three industrialized regions (about $20 \%$ on average), mostly due to postharvest $\mathrm{FaV}$ grading caused by quality standards set by retailers or by international organizations such as the Organisation for Economic Co-operation and Development (OECD) [22] or the United Nations Economic Commission for Europe (UNECE). Waste at the end of the Food Supply Chains (FSC) for FaVs is also substantial in those three regions, with $15-30 \%$ of purchases discarded by consumers mainly in supermarkets and hypermarkets.

In order to reduce this specific case of food waste due to consumers' standard requirements, a recent trend in the retail sector consists of selling "abnormal" (we use this term in the following as a syn-

*firstname.1astname@ec-nantes.fr

†clombart@audencia.com onym of non-standard) $\mathrm{FaVs}$ by emphasizing on pro-environmental considerations. Of course, this trend not only can help reduce food waste but also allows retailers to limit their losses, save money and improve their image.

We chose to focus on $\mathrm{FaVs}$ because they represent one of the 3 "core" departments (namely dairy, meat and produce) in the supermarket distribution sector. Those "core" departments are a real key factor in the choice of a hypermarket or a supermarket by the customers [20]. Indeed, around $80 \%$ of the total purchase of produce [13] are made in supermarkets and hypermarkets and that both for FaVs the most important purchase consideration is the appearance of the product (followed by the price). The reasons of switching are better quality (55\%) and better freshness (52\%) long before lower prices $(31 \%)$. Moreover and $25 \%$ of the customers are ready to switch channels for fresh produce.

We believe that Virtual Reality (VR) could prove a major asset to perform marketing studies with fresh products. By replacing real fresh products with virtual ones we could ensure the repeatability of user studies as well as easily control different aspects of freshness or appearance (e.g., misshaped products) and evaluate the consumer behavior of participants [16]. Indeed, the variability and the perishable nature of fresh products make it very difficult for marketing teams to perform repeatable user studies with a large number of participants. Moreover, the aspect of fresh products quickly deteriorates over time and this is further accelerated by the fact that participants may have to examine the products by manipulating them.

As a consequence, we present a user study which aims at evaluating to what extent customers would react (in terms of perceptions and purchase behavior) to misshaped $\mathrm{FaVs}$ when immersed in a virtual supermarket. The use of VR in this case could help us better understand consumer purchase mechanisms by isolating and controlling different aspects of user studies.

In the remainder of this article, we first present a literature review before presenting our experiment and discussing the results. Finally, we draw conclusions and suggest future work.

\section{BACKGROUND}

In this section, we first present a literature review on marketing studies focusing on consumer shopping and consumption behavior of abnormal food as well as on the use of VR in marketing and nutritional studies.

\subsection{Customer shopping and consumption behavior re- garding abnormal food}

In [17], Loebnitz et al. investigated the impact of oddly shaped food (two fruits and two vegetables) on consumer purchase intentions. Using photographies of real FaVs (apples, lemons, carrots and eggplants) they tested the impact of varying levels of food shape abnormality (normal, moderately abnormal, and extremely abnormal) using a between-subjects experimental design with 964 participants from an online household research panel. They showed that food 
shape abnormalities influence consumers' purchase intentions only if the food deviates extremely from the norm. No differences in purchase intentions emerged for moderately abnormal food.

In a recent research article, de Hooge et al. [11] conducted an online survey where 4214 consumers from five Northern Europe countries revealed their preferences in terms of consumer behavior with respect to imperfect or "abnormal" foods (apples, cucumbers, biscuits, juices, yogurts and milk). Their findings differ greatly depending on demographics, personality characteristics, type of imperfection (in terms of appearance, date labeling, packaging, etc.) or even location of the purchase. This confirms that the mechanisms involved in this phenomenon are very complex and difficult to study.

\subsection{VR in Marketing studies}

VR has been used in relation to marketing in three main ways: (1) as a tool to design and plan shelves inside a supermarket; (2) to perform marketing studies in (desktop) VR environments and (3) in order to compare results obtain in virtual and real supermarkets.

\subsubsection{VR to simulate a real supermarket}

Being able to simulate a store offers a wide range of benefits to conduct studies related to customer behavior. VR provides researchers and professionals with tools that allow for realistically and efficiently recreating virtual supermarkets as well as the flexibility to quickly modify either their layout or the goods presented to the customers.

Concerning off-the-shelf products, Dassault Systèmes offers a software called "Perfect Shelf" [10] that allows for realistically simulating retail settings in an immersive VE. The user can easily design virtual shelves as well as their layout. User studies can then be performed within this software to test retailers' or brand manufacturers' hypotheses. The main limitation with this software is that it only supports packaged goods and therefore cannot be used for the key "fresh departments" (i.e. meat, produce, dairy).

\subsubsection{Performing marketing studies in virtual supermarkets}

Studies using immersive virtual stores in order to investigate consumer perceptions and behavior are rare in the field of marketing and nutrition and mostly rely on desktop VR. Nevertheless, some academic studies should be mentioned.

On the one hand, Pantano and Servidio [23] first investigated how using virtual reality in the points of sale, from the user standpoint, influences consumer perception and satisfaction. They used a virtual fashion clothing store consisting of a large stereo screen. They showed that consumer's satisfaction towards the introduction of immersive virtual environments (VE) in traditional points of sale is influenced by three dimensions: (1) perceived ease of use of the innovative tools, (2) provided enjoyment, and (3) new store perception.

Papagiannidis et al. [24] created their own virtual retail environment consisting of a two-floor shop with fashion clothing for sale. They proposed different models to characterize how participants rate a "quality" criterion (e.g., level of control in the VE, level of realism of the VE, color and graphics "vividness") could be related to their "user experience" (e.g., engagement, enjoyment, pleasure, satisfaction, etc.). They found that level of control, color and graphics vividness as well as 3D authenticity positively affect users' simulated experience which in turn creates higher levels of engagement within the simulated retail environment. They also showed the major impact of satisfaction in purchase intentions.

Wu et al. [36] studied the effect of three virtual fashion clothing stores on 145 consumers' retailer interest, retail pleasure, patronage intention, and purchase behavior. Each store had a different way of grouping the fashion products: a store grouped products by their color (warmness/coolness), a second store grouped products by visual texture (smoothness/thickness) while the third store grouped products by style coordination (clothes that could be worn together in an outfit). They showed that consumers who shopped in the style coordination store spent significantly more money than those who shopped in color or visual texture stores. Those who shopped in the color store experienced significantly more retail pleasure and showed significantly higher patronage intention than those who shopped in the visual texture and style coordination stores, and they showed more retailer interest than consumers in the visual texture store.

On the other hand Waterlander et al. $[32,34]$ indicated that a 3D web-based virtual supermarket can be used to study consumers' reactions to food products' different pricing or labeling strategies. More specifically, in [33], Waterlander et al. performed a study where 115 participants had to purchase products in a 3D web-based virtual supermarket. They evaluated how a $25 \%$ discount on FaVs (63 participants) impacted the number of purchased FaVs compared to normal prices (53 participants). This study was not restricted to fresh FaVs (participants were proposed a wide choice of fresh, frozen and canned $\mathrm{FaVs}$ ) and the $3 \mathrm{D}$ models used for the FaVs were limited to a single model per category (i.e., all carrots were similar). Results show that the $25 \%$ discount led to an increased number of purchased $\mathrm{FaVs}$ while the total number of calories and the expenditure on unhealthy products purchased was similar in both groups, meaning that the money saved with the discount was only used to purchase $\mathrm{FaVs}$.

In the same vein, Massara et al. [18] and van Herpen et al. [28] respectively showed that virtual supermarkets are appropriate to study consumers' reactions to product scarcity as well as consumers' emotional responses while shopping in different environments.

In [19], Nederkoorn et al. used a scenario and a web-based non-virtual (using a picture of a real typical food item per category of product) supermarket to study how hunger and impulse could impact customers purchase of food. 94 participants were given a virtual budget to buy as much food and drinks as they liked in the virtual supermarket for three days. They showed that hungrier and more impulsive participants bought more calories, in particular high caloric snack food.

\subsubsection{Comparisons between real and virtual supermarkets}

When resorting to virtual supermarkets to study consumer's behavior, it is natural to question their comparability with results obtained in a real supermarkets. Bressoud [6] first examined the discrepancy between a real store and a virtual one for a new cereal considering consumer's attitude (measured through its three dimensions: feeling, belief and intention) and behavior (measured by time spent purchasing and purchase rate).

Using a virtual supermarket displayed with three LCD screens, a real physical store and pictures, van Herpen et al. [29] pointed out that consumers tend to buy more products in laboratory conditions than in the actual store due to increased variety seeking. They indicated however that virtual supermarkets do better than the use of pictures to simulate shopping behavior.

Finally, Waterlander et al. [31] recently showed that shopping patterns in a virtual supermarket were comparable to those in real life and conclude that virtual supermarkets are a valid tool to measure food purchasing behavior. It is worth noting that authors stressed that it is important to improve the functionality of some food categories, in particular fruits, vegetables and dairy.

In essence, these academic studies mainly used virtual fashion clothing stores or supermarkets and the vast majority used desktop VR systems. It is worth noting that apart from Waterlander et al. [31,34], none of these research integrated fresh food and more specifically FaVs. Only [33] used some fresh FaVs in their webbased 3D supermarket but without offering any variability (a single 3D model was a used for each type of $\mathrm{FaV}$ ). 


\section{EXPERIMENT}

\subsection{Apparatus and participants}

Participants were sitting comfortably on a chair in front of a desk and immersed in our virtual supermarket using an Oculus Rift DK2 HeadMounted Display (HMD). Given the size of our virtual supermarket $\left(\approx 96 \mathrm{~m}^{2}\right)$ as well as the number of participants (142), we excluded a motion tracking based navigation metaphor and decided to use an Xbox One controller instead. Head-tracking was achieved using the optical camera from the DK2, cf. Fig. 1, and the application ran on a workstation powerful enough to ensure a stable framerate of $75 \mathrm{~Hz}$.

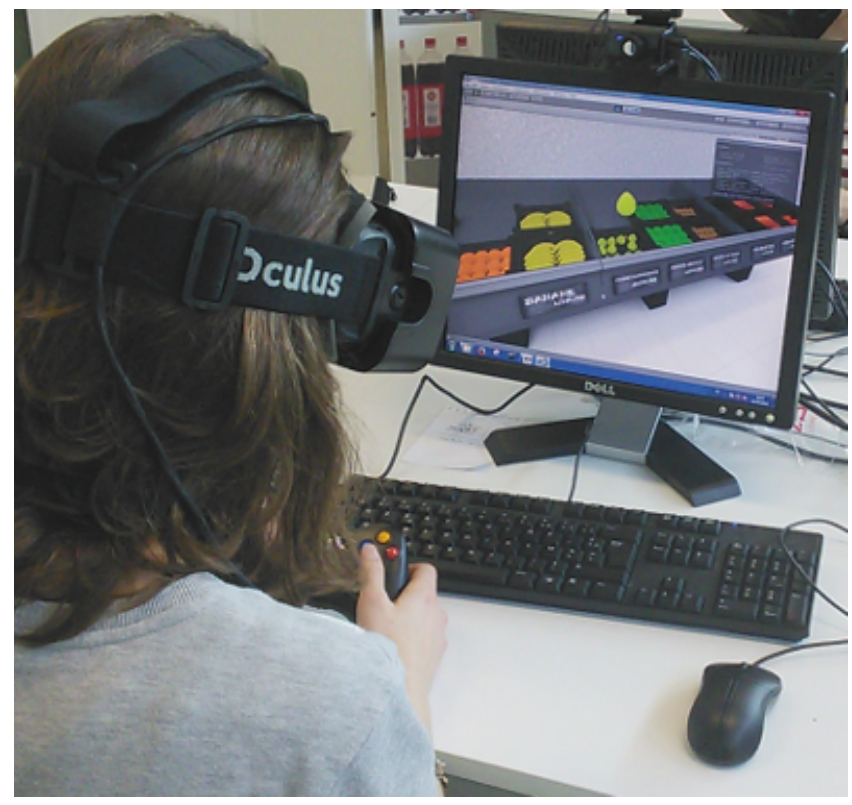

Figure 1: The experimental setup.

142 participants (92 female and 50 male) from a business school took part in the experiment in March 2016. Female participants were aged from 19 to 23 years $(M=20.43, S D=0.7)$ and male were aged from 19 to 22 years $(M=20.36, S D=0.8)$. Of the 142 participants, $103(72.5 \%)$ had no previous experience with virtual reality, 35 (24.6\%) had some previous experience with VR and 4 (2.8\%) declared themselves to be familiar with VR.

We also questioned participants about their food waste consciousness. A total of 95 participants $(66.9 \%)$ declared they would feel ashamed to waste food in front of someone and 114 participants $(80.3 \%)$ declared that avoiding food waste is one of their priorities (they rated at least 5 on a 6-point Likert scale). Moreover 105 participants (74\%) declared that food waste is a major issue in their country and $108(76.1 \%)$ declared that food waste has major consequences for the planet (again giving at least a rate of 5).

We chose an even scale (6-point Likert scale) so that respondents cannot adopt a neutral position considering the subject under study and the bias of social desirability it can imply [9]. This seems reasonable given the topic of our study: food waste has a clear bias towards social acceptance. According to Cialdini's [8] principle of 'Social Proof' people want to behave as the group they belong to or want to belong to, and it is not an acceptable behavior to waste food.

\subsection{The Virtual Supermarket}

\subsubsection{The Virtual Shop and FaVs}

We modeled our virtual supermarket so that that it mimicked $\mathrm{Au}-$ dencia Business School's INSITU store laboratory where marketing studies are carried out on real (non perishable) products.
Our virtual supermarket's furniture as well as non-perishable products (rice, pasta, etc.) were modeled in Blender v2.7 and the scenario was setup under Unity 3D v5.3. The virtual supermarket has a size of about $12 \times 8 \times 2.7 \mathrm{~m}$. It consists of four shelves $(6 \mathrm{~m}$ in length each) filled with non-perishable food products (about 1.000 products per shelf), a produce stand ( $3.4 \mathrm{~m}$ in length) modeled after real-life examples as well as a cashier and a door, cf. Fig. 2. The produce stand contains 8 different types of FaVs stored into 23 baskets. Each basket holds between 12 and 15 items of the same type of FaV, cf. Fig. 5.

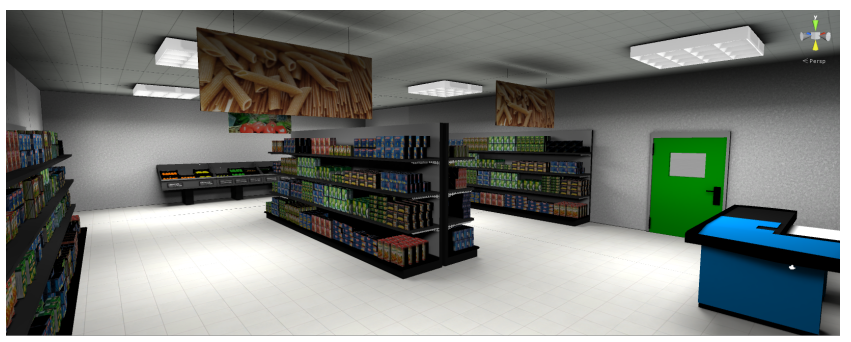

Figure 2: Our Virtual Supermarket (First Person Perspective view).

Concerning the products, we used two different approaches:

- Non-perishable food products were hand-modeled using the products' real dimensions and textured with high resolution pictures taken from the real products.

- FaVs were automatically generated. Since the goal of our study was to test whether customers would buy misshaped $\mathrm{FaVs}$, we needed to have an important number of FaVs of different deformation levels (about $300 \mathrm{FaVs}$ per deformation level). This would have been very time consuming to generate manually. Furthermore, we wanted to have control on the deformation levels as well as to easily be able to generate new sets of FaVs from different levels. A detailed description of our semi-automated $\mathrm{FaV}$ technique is presented in Sect. 7.

Our method generates a wide variety of $\mathrm{FaVs}$ in a semi-automated way based on the following user inputs, cf.Fig. 3:

- A "base" skeleton (composed of a set of 3D points).

- A "base" 2D cross-section of the targeted FaV family (e.g., carrot, apple, etc.).

- An interval and a probability $p_{\text {def }}$ for each skeleton node, representing the range of acceptable deformations as well as the probability $p_{\text {branch }}$ to create new branches in the skeleton.

While our approach was successful to procedurally model misshaped FaVs (cf. Fig. 4), it is not perfect: (1) the generated FaVs are not the result of physiological processes, therefore their deformities are not always physiologically realistic; (2) some of the more heavily deformed FaVs are unrealistic.

Addressing both of these issues in a procedural and automated way is beyond the scope of this paper and would present a real challenge. Indeed, to the best of our knowledge, only very few articles propose a way to procedurally modeled misshaped FaVs [5] and none follows physiological processes.

As a consequence, in order to present our participants with a set of visually realistic FaVs, we performed a manual selection of the automatically generated $\mathrm{FaVs}$ before using them in our virtual supermarket in order to make sure their deformities were visually plausible as well as representative of real misshaped $\mathrm{FaVs}$.

In this study, we used the following 8 families of FaVs (4 fruits and 4 vegetables) in the produce stand (cf. Fig. 2): Oranges, Bananas, Pears (Conference variety), Apples (Granny Smith), Potatoes, Zucchinis, Carrots, (Round) Tomatoes.

Those varieties were chosen because they were seasonal at the time our study was carried out (in March 2016). The only information displayed about the $\mathrm{FaVs}$ was their price which was calculated 


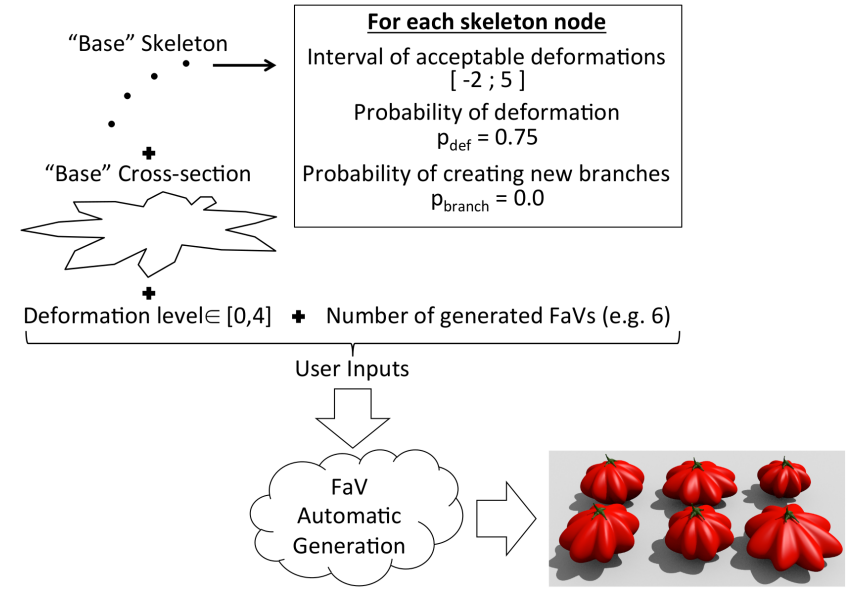

Figure 3: Semi-automated generation of non standard FaVs. Based on a set of user inputs our method generates a set (here 6) of non standard FaVs.

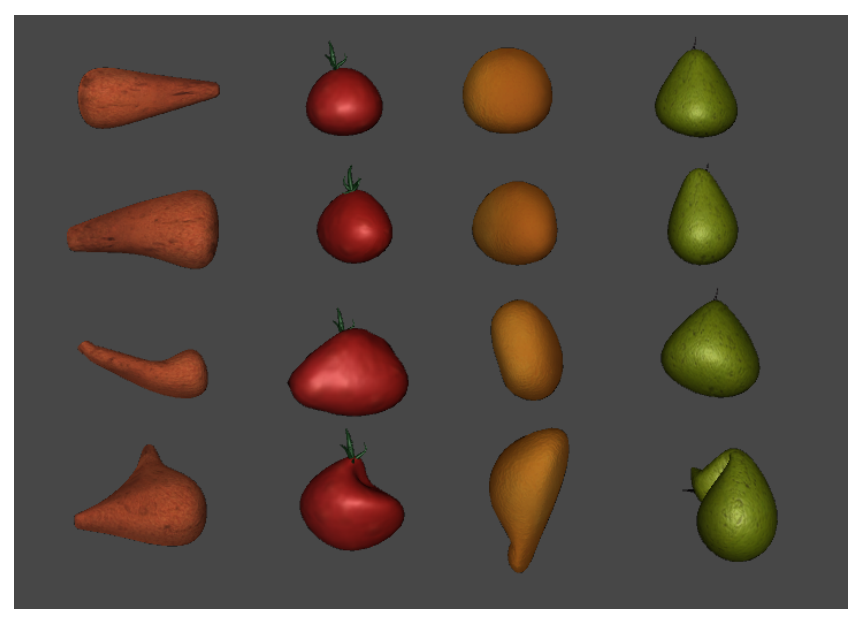

Figure 4: Automated generation (from top to bottom) of a normal, a "slightly" misshaped, a misshaped and a "heavily" misshaped 3D models for vegetables (tomatoes, carrots) and fruits (oranges, pears).

as the average of prices noted in three neighboring hypermarkets and supermarkets.

Finally, after automatically generating FaVs of the 8 families for each one of the 4 conditions (cf. Sect. 3.3.1), we randomly generated between 12 and 15 fruits or vegetables per basket in our produce stand, cf. Fig. 5. We validated that the control condition contained "normal" FaVs, by showing them to consumers and experts in the marketing and nutrition fields.

\subsubsection{Navigation in the VE and manipulation of the virtual FaVs}

The navigation was implemented as follows: the user could move forward and backward using the left thumbstick of the controller. The walking direction was controlled either by the gaze direction (i.e. HMD movements) or by the right thumbstick (in this case the rotation speed was limited in order to reduce dizziness). The walking speed was adjusted to that of a normal real pace.

The user could interact with our virtual objects in a number of different ways, they could: select, manipulate (i.e. rotate), purchase or put back a virtual object. This was achieved using two buttons and one joystick of the controller.

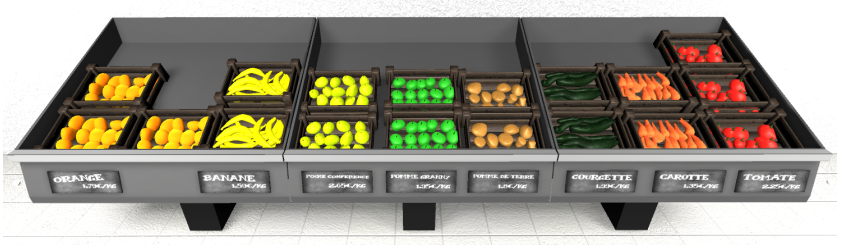

Figure 5: Produce stand filled with automatically generated "normal" FaVs from the 8 families used in our experiment. Below each family of $\mathrm{FaV}$ is a price tag with the name of the FaV and its price per kilo.

The selection mechanism was gaze-based and implemented as follows: a small transparent red dot was displayed (constantly) in the middle of the user's view and served as target for selection. Participants could thus aim with their head movements and could select a product by pressing a button on the controller. Once selected, a bigger self-rotating version of the object appeared in front of them and they could manually rotate the object to inspect it (by using the same thumbstick that adjusts the rotation while navigating). Participants could then either put the object back where at its original position in the VE (by pressing the same button as for selection) or purchase the product by pressing a second button on the controller. Objects that were purchased disappeared from the VE and were stored in an (invisible) virtual cart.

At any time during the experiment, participants could chose to show the content of their virtual cart by pressing a button on the controller (the one used for purchasing when an object is selected). A virtual window with the list of purchased products was then displayed. Each product had its own name (e.g. carrot, apple, etc.) and price, and the total amount of the whole cart was displayed as well. This allowed participants to know the price of each product as well as the current amount spent in the virtual supermarket.

\subsection{Experimental Protocol}

\subsubsection{Experimental Design}

Before starting the experiment, participants had to sign a consent form and fill-in the demographic and customers' habits survey. They were then briefed about the equipment, were told they were going to be immersed in a virtual environment (VE) and it was made clear that they could stop the experiment at any moment if they felt some kind of dizziness or felt bad. Each participant was always supervised by an assistant throughout the experiment to help them with the controller (they cannot see their hands due to the HMD) as well as if they felt bad (dizziness, etc.).

Using a between-subjects design (more specifically, four groups, after-only design), the participants were randomly assigned either to the control group (denoted Group 0 in the following) or one of the three manipulated groups (denoted as groups 1,2 or 3 ), each corresponding to a different aspect for the FaVs:

- Group 0: normal FaVs, 35 participants (control group that corresponds to standards FaVs that are generally sold in hypermarkets and supermarkets);

- Group 1: "slightly" misshaped FaVs, 36 participants;

- Group 2: misshaped FaVs, 35 participants;

- Group 3: "heavily" misshaped FaVs, 36 participants.

In order to make sure the groups were homogeneous, we computed the chi-squared (for the non-metric variable : sex) as well ANOVAs (for the metric variables: age and level of food waste consciousness). The four groups are homogeneous considering sex $\left(\chi^{2}=1.308 ; p=0.727\right)$, age $(F(3,138)=1.083 ; p=0.358)$ and the level of food waste consciousness measured through a 6point Likert scale comprising 6 items $(\min =15, \max =36, M=30.00$, $S D=4.554 ; F(3,138)=0.442 ; p=0.723)$. 
Each participant was then presented with the scenario described in Table 1. If the participant had questions regarding the scenario, he/she was encouraged to ask the assistant.

Once they had finished with the scenario, they performed a training session (cf. Sect. 3.3.2) before starting the real experiment (cf. Sect. 3.3.3). Once the experiment was finished, they were asked to fill-in a post-experimental questionnaire.

\subsubsection{Training session}

A training session was held to have the participants learn how to navigate in the virtual supermarket as well as how to select, manipulate and buy the $\mathrm{FaVs}$.

In this training session, participants were first presented with a text explaining that they were immersed in a virtual supermarket where they could navigate using the controller and that this tutorial would teach them how. A virtual blinking red arrow then appeared in the virtual supermarket and the text directed participants to go underneath it.

Once beneath the arrow, they had to turn in order to face a shelf and were told to select a packet of pasta and, after inspecting it, to put it back on the shelf. To learn how to buy a product, another arrow directed them toward another shelf with packets of rice, one of which they had to buy. They were then told how to display their virtual cart.

Once the purchase was done, participants were told to reach the cashier (highlighted by a big red blinking arrow) in order to finish the training session. This took 3 minutes on average.

\subsubsection{Shopping FaVs in VR}

The task participants had to carry out during the main experiment was described in the experimental scenario (cf. Table 1). Participants were immersed in the virtual supermarket and had to go trough the store (in order to increase the feeling of being in a store) before reaching the produce stand. There, they inspected and choose the FaVs they decided to buy (as mentioned above, they were not forced to buy FaVs if they did not want to).

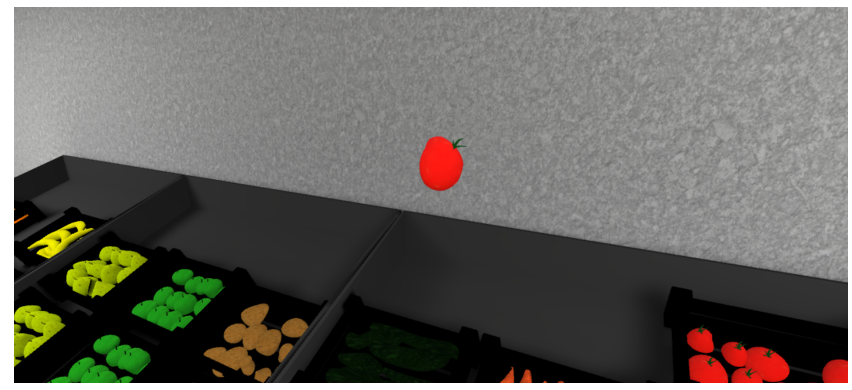

Figure 6: Manipulation of a "misshaped" round tomato (screenshot from the HMD view).

Consumer Behavior Once participants were satisfied with the FaVs they chose, they had to go to the virtual cashier to finish the experiment. For each participant, we recorded the "deformation level" of the FaVs (normal, "slightly" misshaped, misshaped, "heavily" misshaped), the total number of FaVs purchased by family (i.e. fruits or vegetables), the time spent looking at $\mathrm{FaVs}$ per family (fruits or vegetables), as well as the total time taken for each participant were recorded. Moreover we also recorded for each participant the amount of money spent on fruits, on vegetables as well as the total amount spent in the virtual supermarket.

Perception of virtual misshaped FaVs Once the virtual shopping was done, participants removed the HMD, took a moment to rest if they wanted to before going to another PC to answer a 6-point
Likert scale questionnaire about their perceptions of the FaVs they were presented in the experiment.

They had to rate their agreement to the following assertions (each criterion was asked for the fruits and then for the vegetables). Note that following [3] and on the basis of results from two Principle Component Analysis (PCA), we decided to regroup those assertions into three main categories: Impression (items I1 to I6); Quality (items Q1 to Q6); and Price (items P1 and P2). These groups allow for a more global analysis of the participants feelings about our virtual FaVs (see more details in Sect. 4.3):

I1 The color of the fruits (or the vegetables) gives me the impression of high-quality products.

I2 The size of the fruits (or the vegetables) gives me the impression of high-quality products.

I3 The shape of the fruits (or the vegetables) gives me the impression of high-quality products.

I4 The apparent texture of the fruits (or the vegetables) gives me the impression of high-quality products.

I5 The apparent freshness of the fruits (or the vegetables) gives me the impression of high-quality products.

I6 The external appearance of the fruits (or the vegetables) gives me the impression of high-quality products.

Q1 I will be satisfied by the taste of the fruits (or the vegetables).

Q2 I will be satisfied by the nutritional quality of the fruits (or the vegetables).

Q3 I will be satisfied by the sanitary quality of the fruits (or the vegetables).

Q4 I will be satisfied by the ease of preparation of the fruits (or the vegetables).

Q5 I will be satisfied by the environmental impact of the production of the fruits (or the vegetables) (i.e. the production of those fruits (or vegetables) is respectful of the environment).

Q6 I will be satisfied by their global quality.

P1 Prices of the fruits (or the vegetables) are fair.

P2 Prices of the fruits (or the vegetables) are appropriate.

Immersion, Presence and Manipulation Finally, the participants were also asked about their feelings regarding their presence within the virtual supermarket as well as the interaction mechanisms. We used a slightly modified and reduced version of the Presence Questionnaire [35] using a 6-point Likert scale. Indeed the focus of our experiment was not to focus on presence in our virtual supermarket and we tried to keep them focused by avoiding too long questionnaires. We only wanted to assess that participants reported comparable levels of presence and ease of interaction within our virtual supermarket in order to be able to compare their perceptions of our virtual FaVs as well as their consumer behavior.

Again, in order to be able to report results at a higher level, we decided to group the questions into two categories: Interaction (items Int1 to Int8) and Presence (items Pre1 to Pre4):

Int1 Interacting with this virtual supermarket felt natural to me.

Int 2 Moving around this virtual supermarket was natural to me.

Int3 I was able to anticipate the consequences of my movements in this virtual supermarket.

Int 4 I was perfectly able to visually explore this virtual supermarket in an active way.

Int5 The sensation of moving within this virtual supermarket was fully realistic.

Int6 I was perfectly able to observe in details the virtual objects.

Int7 I was able to examine the virtual objects from every angle without any difficulty.

Int8 At the end of the experiment I was perfectly able to move around and interact within this virtual supermarket.

Pre1 In a way I had the feeling that this virtual supermarket surrounded me.

Pre2 I felt present in this virtual supermarket.

Pre3 I was not aware of my real environment. 
For your birthday, your friends offered you a "Dinner@Home" kit.

Next weekend, an amateur cook (from a famous TV show) will come to your place (either Saturday or Sunday evening, the choice is yours) and deliver a dinner (s)he will specially prepare for you and 3 of your friends in her/his workshop/kitchen.

The content of the menu is kept a surprise and the cook is in charge of buying all the products (s)he will use in your menu at her/his usual providers, except for the fruits and vegetables that the cook decided to let up to you. In order for you to do the shopping, the cook gave you a budget of $20 €$ that you can spend entirely or not.

Next to your home, there are two supermarkets: one from the retailer "Super U" and one from another retailer. Today, when passing by, you decide to enter the supermarket owned by the retailer "Super U" and shop your fruits and vegetables there.

For your Dinner@Home, you have to buy at least 2 different types of fruits and at least 2 different types of vegetables at your liking in quantities that you see fit for four people.

According to your own appreciation of the offer of fruits and vegetables in this "Super U"'s shop, you can shop either all the fruits and vegetables here or only part or none of them. Indeed, if need be, you can easily buy some more fruits and vegetables in your neighborhood's other supermarket where you have to go tomorrow anyway to pick up a parcel from an Internet order.

Once your shopping of exclusively fresh fruits and vegetables is done, you will go to the cashier where the goods will be "weighed" and the total amount of your purchase will be displayed.

Finally, we will seek your opinion on the offer of fresh fruits and vegetables in this "Super U"'s shop as well as on the image that this shop and the "Super U" gave you in general.

Table 1: Our experimental scenario, whenever participants had doubts they could ask precisions to the assistants that were here to help them throughout the experiment.

Pre4 I was completely captivated by this virtual supermarket.

Once the questionnaire was filled in, the participants could leave. It took approximately 20 minutes for each participant to perform the whole experiment: 10 minutes to fill out all the questionnaires and the demographic information and another 10 minutes to perform the training and carrying out the experiment in VR.

\section{Results}

In this section, we focus respectively on:

1. immersion (presence and interaction) in the VE;

2. the participants' consumer behavior (number of purchased items and time spent) of misshaped virtual FaVs;

3. the participants' perceptions (impression, quality and price) of misshaped virtual FaVs.

For each subsection, we first computed Cronbach's alpha coefficients to validate the internal consistency of each of our categories (regrouping several items of our questionnaire). Then, we performed analyses of variance (ANOVAs using Bonferroni corrected alpha values) to point out differences among the four conditions studied. Finally, when the ANOVAs revealed significant main effects, we performed bidirectional Student's t-tests for paired comparison of means (following Levene's - two-tailed - tests of equality of variances) for post-hoc analyses.

A Shapiro-Wilk test for normality indicated that while the variables related to VR follow a normal distribution (Presence: $p=$ 0.170 ; and Interaction: $p=0.250)$. On the contrary, variables related to the field of marketing (Impression, Quality and Price) do not follow a normal distribution (same test, $p<0.05$ ). Nevertheless, it should be noted that this is often the case in the marketing field of research where participants notoriously tend to be biased towards favorable answers [1].

\subsection{Presence and Interaction}

In this first subsection, we confirm that participants in our experiment shared a comparable experience in terms of presence in the virtual supermarket and that the interaction mechanisms we proposed (via the Xbox One controller) allowed them to navigate and manipulate objects in a natural and easy way.

The computed Cronbach's alpha coefficients for the Presence (grouping four items) and the Interaction categories (eight items)
Table 2: Mean, Standard Deviation (SD) and computed ANOVAs for the Presence (grouping 4 items, maximum value 24 ) and the Interaction (8 items, maximum value 48 ) categories.

\begin{tabular}{lcccccccccc}
\hline Conditions & \multicolumn{2}{c}{$\mathbf{0}(\mathbf{n}=\mathbf{3 5})$} & \multicolumn{2}{c}{$\mathbf{1}(\mathbf{n}=\mathbf{3 6})$} & \multicolumn{2}{c}{$\mathbf{2}(\mathbf{n}=\mathbf{3 5})$} & \multicolumn{2}{c}{$\mathbf{3}(\mathbf{n}=\mathbf{3 6})$} & \multicolumn{2}{c}{ ANOVA } \\
& Mean & SD & Mean & SD & Mean & SD & Mean & SD & F(3,138) & p \\
\hline Presence & 14.057 & 3.804 & 9.336 & 4.309 & 7.362 & 4.810 & 14.306 & 4.616 & 1.439 & 0.234 \\
\hline Interaction & 24.800 & 8.224 & 24.861 & 12.944 & 28.171 & 15.086 & 26.889 & 8.808 & 1.329 & 0.268 \\
\hline
\end{tabular}

are respectively equal to 0.901 and 0.791 . Since these computed coefficients are above 0.7, they are satisfactory [21]. The ANOVAs did not detect significant differences among the four conditions studied, see Table 2.

As a consequence, we proceed under the assumption that the experiment was comparable for each participant, whatever the condition he/she was assigned to, in terms of presence, navigation within the virtual supermarket and manipulation of the virtual FaVs.

\subsection{Consumer behavior regarding misshaped virtual FaVs: number of purchased items and time of ma- nipulation}

The performed ANOVAs suggested a difference among the four conditions only for the time spent manipulating fruits, see Table 3. Consequently, to confirm this result, we conducted for this specific variable a Levene's test of equality of variances $(F(3,138)=$ $0.219, p=0.641$; the two-tailed hypothesis of equal variances is thus accepted) followed by a Student's t-test for paired comparison of means. These complementary tests highlighted a significant difference between the "heavily" misshaped fruits (i.e. condition 3) and the "slightly" misshaped fruits (i.e. condition 1): bidirectional Student's t-test: $t=-2.800, p=0.007, \mathrm{df}=70$. Participants spent significantly more time manipulating the "heavily" misshaped fruits. By contrast, the differences between the four conditions studied considering time spent manipulating vegetables and the number of fruits or vegetables bought are not significant.

\subsection{Consumer perceptions of misshaped virtual FaVs: Impression, Quality and Price}

The computed Cronbach's alpha coefficients for the three categories under study: Impression (grouping six items), Quality (grouping 
Table 3: Mean, Standard Deviation (SD) and computed ANOVAs for consumer behavior: number of purchased fruits (Nb F) and vegetables $(\mathrm{Nb} \mathrm{V})$ and time spent manipulating fruits (Time $\mathrm{F}$ ) and vegetables (Time V).

\begin{tabular}{ccccccccccc}
\hline Conditions & \multicolumn{2}{c}{$\mathbf{0}(\mathbf{n}=\mathbf{3 5})$} & \multicolumn{2}{c}{$\mathbf{1}(\mathbf{n}=\mathbf{3 6})$} & \multicolumn{2}{c}{$\mathbf{2 ( n = 3 5 )}$} & \multicolumn{2}{c}{$\mathbf{3 ( n = 3 6 )}$} & \multicolumn{2}{c}{ ANOVA } \\
& Mean & SD & Mean & SD & Mean & SD & Mean & SD & $\mathbf{F}(\mathbf{3}, \mathbf{1 3 8})$ & p \\
\hline $\mathrm{Nb} \mathrm{F}$ & 7.857 & 3.069 & 7.556 & 3.938 & 7.343 & 3.834 & 6.889 & 2.692 & 0.503 & 0.681 \\
\hline Time F & 43.203 & 27.198 & 33.135 & 24.429 & 39.957 & 26.697 & 50.061 & 26.803 & 2.575 & 0.056 \\
\hline $\mathrm{Nb} \mathrm{V}$ & 9.886 & 4.013 & 10.139 & 5.027 & 9.657 & 4.935 & 10.361 & 5.389 & 0.139 & 0.936 \\
\hline Time V & 57.954 & 38.425 & 55.690 & 45.004 & 58.024 & 34.857 & 65.166 & 35.010 & 0.409 & 0.746 \\
\hline
\end{tabular}

Table 4: Mean, Standard Deviation (SD) and computed ANOVAs for consumer perceptions on the Impression (6 items, maximum value 36 ), Quality (6 items maximum value 36 ) and Price (2 items, maximum value 12) categories for fruits and vegetables.

\begin{tabular}{lcccccccccc}
\hline Conditions & \multicolumn{2}{c}{$\mathbf{0}(\mathbf{n}=\mathbf{3 5})$} & \multicolumn{2}{c}{$\mathbf{1}(\mathbf{n}=\mathbf{3 6})$} & \multicolumn{2}{c}{$\mathbf{2}(\mathbf{n}=\mathbf{3 5})$} & \multicolumn{2}{c}{$\mathbf{3}(\mathbf{n}=\mathbf{3 6})$} & \multicolumn{2}{c}{ ANOVA } \\
Fruits & Mean & SD & Mean & SD & Mean & SD & Mean & SD & $\mathbf{F}(\mathbf{3}, \mathbf{1 3 8})$ & $\mathbf{p}$ \\
\hline Impression & 20.286 & 7.201 & 18.250 & 7.044 & 20.600 & 6.035 & 16.944 & 6.052 & 2.444 & 0.067 \\
\hline Quality & 23.457 & 5.055 & 20.583 & 6.656 & 24.657 & 4.86 & 20.778 & 6.616 & 4.165 & 0.007 \\
\hline Price & 8.229 & 1.972 & 8.139 & 1.900 & 8.486 & 1.704 & 7.833 & 1.444 & 0.826 & 0.482 \\
\hline Vegetables & Mean & SD & Mean & SD & Mean & SD & Mean & SD & $\mathbf{F}(\mathbf{3}, \mathbf{1 3 8})$ & $\mathbf{p}$ \\
\hline Impression & 20.943 & 7.129 & 18.556 & 6.855 & 21.657 & 6.471 & 17.278 & 6.968 & 3.148 & 0.027 \\
\hline Quality & 22.914 & 5.442 & 19.833 & 6.661 & 24.400 & 5.169 & 19.333 & 6.325 & 5.973 & 0.001 \\
\hline Price & 8.200 & 1.828 & 7.972 & 1.934 & 8.657 & 1.514 & 7.639 & 1.570 & 2.187 & 0.092 \\
\hline
\end{tabular}

six items) and Price (grouping two items), are respectively equal to $0.917,0.912$ and 0.879 for fruits and equal to $0.953,0.924$ and 0.876 for vegetables which are satisfactory. The ANOVAs suggested several differences among the four conditions studied for the different categories except for the consumers' perception of the price of fruits, see Table 4.

Consequently, to confirm these results, we conducted Levene's tests of equality of variances followed by Student's t-tests for paired comparison of means.

For the Impression category all the two-tailed hypotheses of equal variances were accepted. As a consequence, the following significant differences may be highlighted:

- Fruits from condition 0 (control) are perceived as significantly better than those from condition 3 ("heavily" misshaped): $F(3,138)=1.149 ; p=0.287 ; t=2.119, p=0.038, \mathrm{df}=69$.

- Vegetables from condition 0 (control) are also perceived as significantly better than those from condition 3 ("heavily" misshaped): $F(3,138)=0.421 ; p=0.519 ; t=2.191, p=$ $0.032, \mathrm{df}=69$.

- Both fruits and vegetables from condition 2 (misshaped) are perceived as significantly better than those of condition 3 ("heavily" misshaped): respectively $F(3,138)=0.033 ; p=$ $0.857 ; t=2.548, p=0.013, \mathrm{df}=69$ and $F(3,138)=$ $0.195 ; p=0.660 ; t=2.742, p=0.008, \mathrm{df}=69$.

As for the Quality category, the significant differences are:

- Both fruits and vegetables from condition 0 (control) are perceived as significantly better than those from condition 1 ("slightly" misshaped): respectively $F(3,138)=2.473 ; p=$ $0.120 ; t=2.044, p=0.045, \mathrm{df}=69$ and $F(3,138)=$ $0.976 ; p=0.327 ; t=2.131, p=0.037, \mathrm{df}=69$.

- Vegetables from condition 0 (control) are also perceived as significantly better than those from condition 3 ("heavily" misshaped): $F(3,138)=1.316 ; p=0.255 ; t=2.554, p=$ $0.013, \mathrm{df}=69$.

- Both fruits and vegetables from condition 1 ("slightly" misshaped) are perceived as significantly better than those of condition 2 (misshaped): respectively $F(3,138)=3.212 ; p=$ $0.077 ; t=-2.938, p=0.004, \mathrm{df}=69$ and $F(3,138)=$ $1.837 ; p=0.180 ; t=-3.221, p=0.002, \mathrm{df}=69$.

- Both fruits and vegetables from condition 2 (misshaped) are perceived as significantly better than those of condition 3 ("heavily" misshaped): respectively $F(3,138)=3.251$; $p=$
$0.076 ; t=2.809, p=0.006, \mathrm{df}=69$ and $F(3,138)=$ 2,$440 ; p=0.123 ; t=3.690, p-$ value $=0.000, \mathrm{df}=69$.

Finally, regarding the Price category, a significant difference exists for vegetables between condition 2 (misshaped) and condition 3 ("heavily" misshaped) that are perceived as significantly better: $F(3,138)=0.041 ; p=0.841 ; t=2.781, p=0.007, \mathrm{df}=69$.

\section{Discussion}

First of all, we showed that there is no significant difference in the mean number of $\mathrm{FaVs}$ bought by our participants whatever the deformation level of those FaVs. This is interesting especially, when compared to results from Loebnitz et al. [17] who found out that participants purchased significantly less $\mathrm{FaVs}$ when they were extremely misshaped. This difference could be due to differences in how participants were presented with the products: photographies of one single real FaV for Loebnitz et al. [17] while we presented them with several 3D virtual products they could manipulate.

This could also have been caused by our experimental scenario: it could have biased participants into buying FaVs. Yet, we clearly noted in the scenario that they did not have to buy FaVs if they did not feel like it, and we clarified it whenever they asked questions. A possible way to overcome this limitations might have been to ask them to shop all the ingredients for the dinner and not only the FaVs.

We also noted that there was only one significant difference when comparing prices of the FaVs (see Table 4), where misshaped vegetables (condition 2), are perceived as having a significantly fairer price compared to vegetables "heavily misshaped" (condition 3 ).

Another finding is that $\mathrm{FaVs}$ of conditions 1 ("slightly" misshaped) and 3 ("heavily" misshaped) are significantly perceived as less qualitative $(p \leq 0.01)$ than those of condition 2 (misshaped). This is interesting since $\mathrm{FaVs}$ in condition 2 were more misshaped than those in condition 1 and because the quality was assessed by a mix of different criteria (color, texture, etc., see Sect. 3.3.3) but not by shape only. When taken individually we also noted than $\mathrm{FaVs}$ from conditions 1 and 3 were all rated lower in those criteria than those in condition 2 (even if not all differences were significant).

Given these results, we propose the hypothesis that $\mathrm{FaVs}$ from condition 3 were too misshaped to be perceived as of quality and that it provoked participants to rate them as lower in all the criteria (and not only the shape). Of course, this could have been caused by our semi-automatic $\mathrm{FaV}$ generation mechanism which produced really misshaped FaVs, as illustrated in Fig. 4. Nevertheless, we compared to the real pictures used in [17] as extremely abnormal $\mathrm{FaVs}$, our "heavily" misshaped FaVs did not seem unrealistic. It would prove beneficial to be able to compare results using both real and $3 \mathrm{D}$ virtual $\mathrm{FaVs}$.

Finally, one could wonder why we focused on non standards FaVs only (i.e. misshaped) while VR could also be used to simulate other phenomena such as decay of FaVs. We chose to study abnormal FaVs since decaying or rotting FaVs cannot be sold in supermarkets: either by law enforcement for advanced decay or by the retailers for moderate decay since it could drive their customers away (the produce department being one of the main reasons customers may chose a new supermarket).

\section{CONCLUSION/FUTURE WORK}

The idea behind this paper was that VR could be used as a tool to address marketing issues on products and in conditions that are difficult to tackle in reality. As a first study, we focused on fresh products, namely fruits and vegetables, which are both a very interesting and a very complex topic for marketing studies. Indeed, FaVs are central for customers' choice of shops, generate huge amount of money for retailers but simultaneously are source of a lot of waste (and thus a loss of money). Fresh products are difficult to study since they vary and deteriorate over time preventing participants to manipulate them. VR has already been used for marketing studies 
but so far, it has been limited to either non-perishable, packaged content or to non realistic fresh content (e.g., by relying on a single 3D model for a family of FaVs). We believe VR could be used to study fresh and perishable products which aspect vary greatly (as individual products and over time).

As a consequence, as a first step towards the use of immersive VR for marketing studies on fresh products, we conducted an experiment studying how customers perceive and to which extent they would be ready to buy "abnormal" FaVs in a virtual supermarket. Users were presented eight families of FaVs from one of four categories of abnormalities: "normal", "slightly misshaped", "misshaped" and "heavily misshaped".

Our results show that there is no significant difference between the mean number of $\mathrm{FaVs}$ purchased by our participants whatever the deformation level. Nevertheless differences were noted between FaVs of different deformation levels regarding their perceived "Quality" (grouping 6 items).

If we had chosen a within-subjects design (one participant would have done all the conditions) instead of our between-subjects one, each participant could have noticed differences between the four conditions in a stronger way. Nevertheless, given the number of subjects as well as conditions, performing a within-subjects experimental design would have required much more time (or less conditions) since we should have left at least a couple of weeks between the different conditions for the same participant.

In order to be able to validate our hypothesis that immersive VR is a promising tool to perform marketing studies that are difficult to realize in real life, our future work consists of performing an experiment where participants are presented real and virtual "abnormal" $\mathrm{FaVs}$ in order to compare their consumer behavior in the real and virtual case. This would help us understand the potential influences of display (e.g., HMD vs. a screen), rendering (colors and textures of $\mathrm{FaVs}$ ) and interaction mechanism (i.e., being able to offer more "natural" interactions with virtual FaVs) on consumers behavior.

We also plan on studying classical marketing leveraging effects such as labeling conditions of price, origin of products (local vs. non local) and method of production (organic vs. non organic) in order to see ho they affect participants' consumer behavior.

\section{ApPENDIX: SEMI-AUtOMATEd GENERATION OF NON STANDARDS FAVS}

We rely on Generalized Cylinders [2,25] (GCs) techniques to generate our FaVs. GCs have been extensively used in procedural and/or organic modeling (e.g., trees [4], plants [14], plants root [27], etc.) and are well-suited to generate the FaVs we are interested in. Other methods could have been used to procedurally model FaVs, such as: custom L-systems $[5,15]$, implicit surfaces or volumetric modeling [30]; but we favored GCs since our FaVs all have cylindrical shapes and were therefore adapted to be generated using GCs techniques. While the purpose of our paper is not to present a method to automatically generate a variety of misshaped $\mathrm{FaVs}$, we provide below an overview of our solution.

In order to generate semi-automatically a set of $\mathrm{FaVs}$ with different deformation levels, we follow a two-step approach:

1. A user generates a "base" model for each type of FaV.

2. This "base" model is automatically modified to generate a wide variety of FaVs for each desired deformation level.

For the first step, the user has to manually create a "base" FaV (cf. Fig. 3 and Fig. 7, left) by defining:

- A 3D skeleton by positioning a set of 3D points, each one being a node of the skeleton.

- A 2D cross-section representing the outline of the $\mathrm{FaV}$, which will later swept along the branches of the skeleton.

- The following variation intervals for each skeleton node:

- The intensity and range of a change in the node's position and orientation.

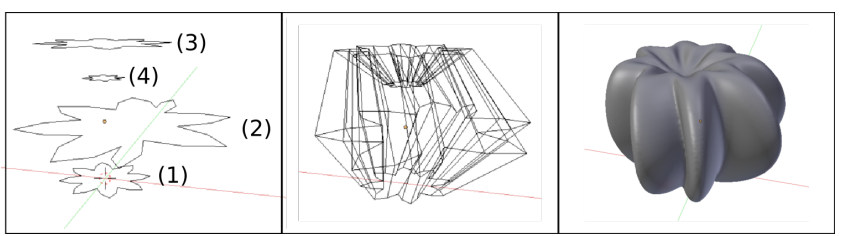

Figure 7: Semi-automated generation of a Beefsteak tomato. Left: Representation of the user-defined $2 \mathrm{D}$ cross-section scaled at each skeletal nodes (the skeleton is composed of 4 nodes along a vertical line). Middle: After cross-section sweeping along the skeleton. Right: Resulting smoothed Beefsteak tomato.

- The intensity and range of a modification in the sweeping cross-section (e.g. scaling the cross-section).

- How likely a node can give rise to a new node in the skeleton (allowing us to later generate a wider range of misshaped $\mathrm{FaVs}$, such as forked carrots).

Note that the intensities and ranges of those variations are directly linked to the desired deformation level of the FaVs.

Once we have for each "base" $\mathrm{FaV}$ its skeleton and cross-section, we proceed as follows to generate a set of deformed FaVs (cf. Fig. 7, middle and right):

- For each skeleton node, variations are randomly applied within the user-defined intervals.

- The cross section is swept (i.e., extruded) along the skeleton.

- The resulting 3D object is finally smoothed (with a CatmullClark subdivision surface [7] and a Laplacian smoothing [26]) to obtain a more organic aspect.

\section{REFERENCES}

[1] D. A. Aaker, V. Kumar, R. Leone, and G. Day. Marketing Research. Wiley, 11 ed., 2012.

[2] G. J. Agin and T. O. Binford. Computer description of curved objects. IEEE Transactions on Computers, 25(4):439-449, apr 1976. doi: 10. 1109/TC. 1976.1674626

[3] P. Aurier and L. Siriex. Marketing des produits agroalimentaires. Dunod, 2009.

[4] J. Bloomenthal. Modeling the mighty maple. ACM SIGGRAPH Computer Graphics, 19(3):305-311, 1985. doi: 10.1145/325165.325249

[5] E. Bohl, O. Terraz, and D. Ghazanfarpour. Modeling fruits and their internal structure using parametric 3Gmap L-systems. The Visual Computer, 31(6-8):819-829, jun 2015. doi: 10.1007/s00371-015-1108 $-9$

[6] E. Bressoud. Testing FMCG innovations: experimental real store versus virtual. Journal of Product \& Brand Management, 22(4):286292, 2013. doi: 10.1108/JPBM-05-2012-0141

[7] E. Catmull and J. Clark. Recursively generated B-spline surfaces on arbitrary topological meshes. Computer-Aided Design, 10(6):350-355, nov 1978. doi: 10.1016/0010-4485(78)90110-0

[8] R. Cialdini. Influence: The Psychology of Persuasion. Harper Business, 2007.

[9] E. P. Cox. The optimal number of response alternatives for a scale: A review. Journal of Marketing Research, 12(4):407-422, nov 1980.

[10] Dassault Systèmes. Perfect Shelf. http://www.3ds.com/ industries/consumer-packaged-goods-retail/perfectshelf/. Accessed: 2016-05-25.

[11] I. E. de Hooge, M. Oostindjer, J. Aschemann-Witzel, A. Normann, S. M. Loose, and V. L. Almli. This apple is too ugly for me!: Consumer preferences for suboptimal food products in the supermarket and at home. Food Quality and Preference, 56, Part A:80-92, mar 2017. doi: 10.1016/j.foodqual.2016.09.012

[12] Food and Agriculture Organization of the United Nations. Global food losses and food waste. http://www.fao.org/docrep/014/mb060e/ mb060e.pdf, 2011. Accessed: 2016-05-19.

[13] Food Marketing Institute. The power of produce, 2015. 
[14] M. Fuhrer, H. W. Jensen, and P. Prusinkiewicz. Modeling hairy plants. Graphical Models, 68(4):333-342, jul 2006. doi: 10.1016/j.gmod. 2005.11.002

[15] C. Y. Huang, W. T. Jheng, W. K. Tai, C. C. Chang, and D. L. Way. Procedural grape bunch modeling. Computers \& Graphics, 37(4):225237, jun 2013. doi: 10.1016/j.cag.2013.01.002

[16] In-Store Marketing Institute and Advertising Research Foundation and Indiana University. Kelley School of Business. Shaping retail: The use of virtual store simulations in marketing research and beyond. http://kelley.iu.edu/cerr/files/ 09ismi_virtualretailing.pdf, 2009. Accessed: 2016-11-29.

[17] N. Loebnitz, G. Schuitema, and K. G. Grunert. Who buys oddly shaped food and why? Impacts of food shape abnormality and organic labeling on purchase intentions. Psychology \& Marketing, 32(4):408-421, apr 2015. doi: 10.1002/mar. 20788

[18] F. Massara, S. S. Liu, and R. D. Melara. Adapting to a retail environment: Modeling consumer-environment interactions. Journal of Business Research, 63(7):673-681, jul 2010. doi: 10.1016/j.jbusres. 2009.05.004

[19] C. Nederkoorn, R. Guerrieri, R. C. Havermans, A. Roefs, and A. Jansen. The interactive effect of hunger and impulsivity on food intake and purchase in a virtual supermarket. International Journal of Obesity, 33(8):905-912, june 2009. doi: 10.1038/ijo.2009.98

[20] Nielsen. Think smaller for big growth: How to thrive in the new retail landscape, june 2016.

[21] J. C. Nunnally. Psychometric Theory. McGraw-Hill, 2 ed., 1978.

[22] Organisation for Economic Co-operation and Development. OECD fruit and vegetables standards: Brochures. http://www.oecd.org/ tad/code/oecdfruitandvegetablesstandardsbrochures.htm, 2010. Accessed: 2016-05-19.

[23] E. Pantano and R. Servidio. Modeling innovative points of sales through virtual and immersive technologies. Journal of Retailing and Consumer Services, 19(3):279-286, may 2012. doi: 10.1016/j. jretconser.2012.02.002

[24] S. Papagiannidis, E. Pantano, E. W. K. See-To, and M. Bourlakis. Modelling the determinants of a simulated experience in a virtual retail store and users product purchasing intentions. Journal of Marketing Management, 29:1462-1492, sep 2013. doi: 10.1080/0267257X.2013. 821150

[25] U. Shani and D. H. Ballard. Splines as embeddings for generalized cylinders. Computer Vision, Graphics, and Image Processing, 27(2):129-156, aug 1984. doi: 10.1016/s0734-189x(84)80039-0

[26] G. Taubin. A signal processing approach to fair surface design. In Proc. SIGGRAPH, pp. 351-358. ACM Press, New York, New York, USA, 1995. doi: 10.1145/218380.218473

[27] X. Tian, G. Han, M. Chen, and Z. Situ. Skeleton-based surface reconstruction for visualizing plant roots. In Proc. ICAT, pp. 328-332. IEEE, 2006. doi: 10.1109/ICAT.2006.117

[28] E. van Herpen, R. Pieters, and M. Zeelenberg. When demand accelerates demand: Trailing the bandwagon. Journal of Consumer Psychology, 19(3):302-312, jul 2009. doi: 10.1016/j.jcps.2009.01.001

[29] E. van Herpen, T. Yu, E. van den Broek, and H. van Trijp. Using a virtual grocery store to simulate shopping behaviour. In Proc. Measuring Behavior, 2014.

[30] L. Wang, Y. Yu, K. Zhou, and B. Guo. Multiscale vector volumes. ACM Transactions on Graphics, 30(6):1, dec 2011. doi: 10.1145/2070781. 2024201

[31] W. E. Waterlander, Y. Jiang, I. H. Steenhuis, and C. Ni Mhurchu. Using a 3D virtual supermarket to measure food purchase behavior: A validation study. Journal of Medical Internet Research, 17(4):e107, Apr 2015. doi: 10.2196/jmir.3774

[32] W. E. Waterlander, M. Scarpa, D. Lentz, and I. H. Steenhuis. The virtual supermarket: An innovative research tool to study consumer food purchasing behaviour. BMC Public Health, 11(1):1-10, jul 2011. doi: 10.1186/1471-2458-11-589

[33] W. E. Waterlander, I. H. Steenhuis, M. R. de Boer, A. J. Schuit, and J. C. Seidell. The effects of a $25 \%$ discount on fruits and vegetables: results of a randomized trial in a three-dimensional web-based supermarket. International Journal of Behavioral Nutrition and Physical Activity, 9(1):11, feb 2012. doi: 10.1186/1479-5868-9-11
[34] W. E. Waterlander, I. H. Steenhuis, M. R. de Boer, A. J. Schuit, and J. C. Seidell. Effects of different discount levels on healthy products coupled with a healthy choice label, special offer label or both: results from a web-based supermarket experiment. International Journal of Behavioral Nutrition and Physical Activity, 10(1):59, may 2013. doi: 10.1186/1479-5868-10-59

[35] B. G. Witmer, C. J. Jerome, and M. J. Singer. The factor structure of the presence questionnaire. Presence: Teleoperators and Virtual Environments, 14(3):298-312, 2005. doi: 10.1162/105474605323384654

[36] J. Wu, H. W. Ju, J. Kim, C. Damminga, H.-Y. Kim, and K. Johnson. Fashion product display: An experiment with mockshop investigating colour, visual texture, and style coordination. International Journal of Retail \& Distribution Management, 41(10):765-789, oct 2013. doi: 10. 1108/IJRDM-08-2012-0072 